\title{
CAMA
}

Centre for Applied Macroeconomic Analysis

\section{Modeling energy price dynamics: GARCH versus stochastic volatility}

\section{CAMA Working Paper 20/2015 June 2015}

\author{
Joshua C.C. Chan
}

Research School of Economics, ANU and

Centre for Applied Macroeconomic Analysis (CAMA), ANU

\section{Angelia L. Grant}

Research School of Economics, ANU

\begin{abstract}
We compare a number of GARCH and stochastic volatility (SV) models using nine series of oil, petroleum product and natural gas prices in a formal Bayesian model comparison exercise. The competing models include the standard models of $\operatorname{GARCH}(1,1)$ and SV with an $A R(1)$ log-volatility process and more flexible models with jumps, volatility in mean and moving average innovations. We find that: (1) SV models generally compare favorably to their GARCH counterparts; (2) the jump component substantially improves the performance of the standard GARCH, but is unimportant for the SV model; (3) the volatility feedback channel seems to be superfluous; and (4) the moving average component markedly improves the fit of both GARCH and SV models. Overall, the SV model with moving average innovations is the best model for all nine series.
\end{abstract}




\section{Keywords}

Bayesian model comparison, crude oil, natural gas, moving average, jumps

\section{JEL Classification}

C11, C52, Q41

Address for correspondence:

(E) cama.admin@anu.edu.au

The Centre for Applied Macroeconomic Analysis in the Crawford School of Public Policy has been established to build strong links between professional macroeconomists. It provides a forum for quality macroeconomic research and discussion of policy issues between academia, government and the private sector.

The Crawford School of Public Policy is the Australian National University's public policy school, serving and influencing Australia, Asia and the Pacific through advanced policy research, graduate and executive education, and policy impact. 


\title{
Modeling Energy Price Dynamics: GARCH versus Stochastic Volatility
}

\author{
Joshua C.C. Chan* Angelia L. Grant \\ Research School of Economics, \\ and Centre for Applied Macroeconomic Analysis, \\ Australian National University
}

June 2015

\begin{abstract}
We compare a number of GARCH and stochastic volatility (SV) models using nine series of oil, petroleum product and natural gas prices in a formal Bayesian model comparison exercise. The competing models include the standard models of $\operatorname{GARCH}(1,1)$ and $\mathrm{SV}$ with an $\mathrm{AR}(1)$ log-volatility process and more flexible models with jumps, volatility in mean and moving average innovations. We find that: (1) SV models generally compare favorably to their GARCH counterparts; (2) the jump component substantially improves the performance of the standard GARCH, but is unimportant for the SV model; (3) the volatility feedback channel seems to be superfluous; and (4) the moving average component markedly improves the fit of both GARCH and SV models. Overall, the SV model with moving average innovations is the best model for all nine series.
\end{abstract}

Keywords: Bayesian model comparison, crude oil, natural gas, moving average, jumps.

JEL classification: C11, C52, Q41

*Financial support from the Australian Research Council via a Discovery Early Career Researcher Award (DE150100795) is gratefully acknowledged. 


\section{Introduction}

The volatility of oil, petroleum product and natural gas prices has broad economic and financial implications, and this has motivated a vast literature on modeling such volatility. Conventionally, this time-varying volatility is modeled - and the fit assessed - using various generalized autoregressive conditional heteroscedastic (GARCH) models, under which the conditional variance is a deterministic function of model parameters and past data (see, e.g., Fong and See, 2001; Sadorsky, 2006; Kang, Kang, and Yoon, 2009; Agnolucci, 2009; Mohammadi and Su, 2010; Nomikos and Andriosopoulos, 2012; Mason and Wilmot, 2014; Manera, Nicolini, and Vignati, 2014).

Alternatively, some recent papers have considered stochastic volatility models, where the volatility is a latent variable that follows a stochastic process (see, e.g., Sadorsky, 2005; Vo, 2009; Trolle and Schwartz, 2009; Larsson and Nossman, 2011; Brooks and Prokopczuk, 2013). These two classes of models are nonnested and the implied time-varying volatilities have very different properties. To the extent that they are compared at all, the literature has mainly focused on their forecasting performance. While volatility forecasting is an important problem, energy prices are widely used in macroeconomic models to analyze the interplay between these prices and the macroeconomy (see, e.g., Kilian, 2009; Peersman and Van Robays, 2012; Blanchard and Riggi, 2013). Consequently, it is of interest to directly compare the model fit of these two classes of time-varying volatilty models in a formal model comparison exercise, but this is rarely done in practice.

We fill this gap by assessing the model fit - while penalizing model complexity - of a number of GARCH and stochastic volatility models for modeling the dynamics of oil, petroleum product and natural gas prices. To that end, we perform a formal Bayesian model comparison exercise to assess the evidence in favor of the GARCH and stochastic volatility models given the data. Specifically, for each model we compute its marginal data density, which evaluates how likely it is for the observed data to have occurred given the model. Using this measure we can further obtain the posterior probabilities of the models (see, e.g., Koop, 2003, for a detailed discussion on Bayesian model comparison).

For the model comparison exercise, we consider five commonly-used GARCH models in the literature: the standard $\operatorname{GARCH}(1,1)$ model, and the more flexible models of $\operatorname{GARCH}(2,1)$, GARCH with jumps, GARCH in mean and GARCH with moving average innovations. We then choose five stochastic volatility models that are close counterparts of these GARCH models. By directly comparing the GARCH and stochastic volatility mod- 
els pairwise, we can assess whether the deterministic conditional heteroscedasticity under GARCH or the stochastic variance under SV is more favored by the data. In addition, we can compare the more flexible GARCH variants against the standard GARCH - and the flexible stochastic volatility models against the standard SV-to examine what features are most empirically relevant for energy prices, thus providing useful and practical guidelines for practitioners.

The main results can be summarized as follows. First, the stochastic volatility models generally compare favorably to their GARCH counterparts, indicating that the timevarying volatility is better modeled as a latent stochastic process. This result is in line with the finding in the finance literature that stochastic volatility models often fit financial returns better (see, e.g., Kim, Shepherd, and Chib, 1998; Yu, 2002).

Second, the jump component substantially improves the performance of the standard GARCH, but is unimportant for the stochastic volatility model. Since the standard GARCH specifies a deterministic conditional variance process, adding a random jump component appears to give the model additional flexibility against misspecification. This is apparently unnecessary for the stochastic volatility model. Third, the volatility feedback channel in both the GARCH in mean and stochastic volatility in mean models seems to be superfluous. Fourth, the moving average component substantially improves the fit of both types of models. In fact, the stochastic volatility model with moving average innovations is the best overall model for all the energy prices considered.

The rest of this article is organized as follows. Section 2 introduces the two classes of timevarying volatility models - GARCH and stochastic volatility models. In Section 3 we give an overview of Bayesian model comparison and outline an adaptive importance sampling approach to compute the marginal likelihood for comparing models. Section 4 compares the performance of the GARCH models with their stochastic volatility counterparts. Estimation results are also reported. Lastly, Section 5 concludes and briefly discusses some future research directions.

\section{Time-Varying Volatility Models}

In this section we discuss the two classes of time-varying volatility models used in the model comparison exercise. The first class of models is the generalized autoregressive conditional heteroskedastic (GARCH) models, which are developed by Bollerslev (1986) 
to extend the earlier work on ARCH models by Engle (1982). The second set of models is the stochastic volatility models, which are considered by Taylor (1994). In these models the volatility is specified as a latent stochastic process.

\section{$2.1 \quad$ GARCH Models}

In this section we describe various GARCH models that are widely used to model energy prices. The first one is the standard $\operatorname{GARCH}(1,1)$ model, which we simply refer to as GARCH:

$$
\begin{aligned}
y_{t} & =\mu+\varepsilon_{t}, & \varepsilon_{t} \sim \mathcal{N}\left(0, \sigma_{t}^{2}\right), \\
\sigma_{t}^{2} & =\alpha_{0}+\alpha_{1} \varepsilon_{t-1}^{2}+\beta_{1} \sigma_{t-1}^{2}, &
\end{aligned}
$$

where $\varepsilon_{0}=0$ and $\sigma_{0}^{2}$ is a constant. To ensure the variance process $\sigma_{t}^{2}$ is always straightly positive and stationary, we assume that $\alpha_{0}>0, \alpha_{1} \geqslant 0, \beta_{1} \geqslant 0$ and $\alpha_{1}+\beta_{1}<1$. Note that the conditional variance $\sigma_{t}^{2}$ is a deterministic function of the model parameters and past data.

The conditional variance $\sigma_{t}^{2}$ in (2) follows an $\mathrm{AR}(1)$ process. Next, we consider the $\operatorname{GARCH}(2,1)$ model - in which $\sigma_{t}^{2}$ follows an $\mathrm{AR}(2)$ process - that allows for richer variance dynamics:

$$
\sigma_{t}^{2}=\alpha_{0}+\alpha_{1} \varepsilon_{t-1}^{2}+\beta_{1} \sigma_{t-1}^{2}+\beta_{2} \sigma_{t-2}^{2},
$$

where $\sigma_{-1}^{2}=\varepsilon_{0}=0$ and $\sigma_{0}^{2}$ is a constant. Again, to ensure the variance process $\sigma_{t}^{2}$ is always straightly positive and stationary, we assume that the parameters $\alpha_{0}, \alpha_{1}, \beta_{1}$, and $\beta_{2}$ are all positive and $\alpha_{1}+\beta_{1}+\beta_{2}<1$. We refer to this model as GARCH-2.

The third GARCH model allows for the possibility of infrequent "jumps" in the data series, which can accommodate drastic changes in energy prices. More specifically, consider the following GARCH with jumps (GARCH-J) model:

$$
\begin{array}{rlr}
y_{t} & =\mu+k_{t} q_{t}+\varepsilon_{t}, & \varepsilon_{t} \sim \mathcal{N}\left(0, \sigma_{t}^{2}\right), \\
\sigma_{t}^{2} & =\alpha_{0}+\alpha_{1}\left(y_{t-1}-\mu\right)^{2}+\beta_{1} \sigma_{t-1}^{2}, &
\end{array}
$$

where $q_{t} \in\{0,1\}$ is a jump variable with success probability $\mathbb{P}\left(q_{t}=1\right)=\kappa$. Hence, if $q_{t}=1$, a jump occurs at time $t$ and its size is determined by $k_{t}$, which is modeled as $k_{t} \sim \mathcal{N}\left(\mu_{k}, \sigma_{k}^{2}\right)$. 
Next, consider the GARCH in mean (GARCH-M) model, under which the conditional variance enters the conditional mean as a covariate:

$$
\begin{aligned}
y_{t} & =\mu+\lambda \sigma_{t}^{2}+\varepsilon_{t}, & \varepsilon_{t} \sim \mathcal{N}\left(0, \sigma_{t}^{2}\right) \\
\sigma_{t}^{2} & =\alpha_{0}+\alpha_{1} \varepsilon_{t-1}^{2}+\beta_{1} \sigma_{t-1}^{2} . &
\end{aligned}
$$

This variant allows for the possibility that the data series depends on its volatility (risk). It is obvious that when $\lambda=0$, the GARCH-M model reduces to the GARCH model.

Lastly, we combine a first-order moving average model with GARCH innovations:

$$
\begin{aligned}
& y_{t}=\mu+\varepsilon_{t}, \\
& \varepsilon_{t}=u_{t}+\psi u_{t-1}, \quad u_{t} \sim \mathcal{N}\left(0, \sigma_{t}^{2}\right),
\end{aligned}
$$

where the invertibility condition is imposed, i.e., $|\psi|<1$. The variance $\sigma_{t}^{2}$ again follows the same GARCH process as in (2). This GARCH model is referred to as GARCH-MA. In contrast to the other GARCH models considered above, this model allows the data series to be correlated over time and might better model the short-run dynamics of the series.

\subsection{Stochastic Volatility Models}

Next we introduce the five stochastic volatility models that are close counterparts of the GARCH models described in the previous section. The volatility under a stochastic volatility model is a random variable, in stark contrast to GARCH models in which the conditional variance is a deterministic function of the model parameters and past data.

The first model is the standard stochastic volatility (SV) model:

$$
\begin{array}{llrl}
y_{t}=\mu+\varepsilon_{t}^{y}, & \varepsilon_{t}^{y} \sim \mathcal{N}\left(0, \mathrm{e}^{h_{t}}\right), \\
h_{t}=\mu_{h}+\phi_{h}\left(h_{t-1}-\mu_{h}\right)+\varepsilon_{t}^{h}, & \varepsilon_{t}^{h} \sim \mathcal{N}\left(0, \omega_{h}^{2}\right) .
\end{array}
$$

The log-volatility $h_{t}$ follows a stationary $\mathrm{AR}(1)$ process with $\left|\phi_{h}\right|<1$ and unconditional mean $\mu_{h}$. The process is initialized with $h_{1} \sim \mathcal{N}\left(\mu_{h}, \omega_{h}^{2} /\left(1-\phi_{h}^{2}\right)\right)$.

In the second stochastic volatility model, the observation equation is the same as in (3), 
but the log-volatility $h_{t}$ now follows a stationary $\operatorname{AR}(2)$ process:

$$
h_{t}=\mu_{h}+\phi_{h}\left(h_{t-1}-\mu_{h}\right)+\rho_{h}\left(h_{t-2}-\mu_{h}\right)+\varepsilon_{t}^{h}, \quad \varepsilon_{t}^{h} \sim \mathcal{N}\left(0, \omega_{h}^{2}\right),
$$

where we assume the roots of the characteristic polynomial associated with $\left(\phi_{h}, \rho_{h}\right)$ lie outside the unit circle. Further, $h_{1}$ and $h_{2}$ are assumed to follow the unconditional distribution:

$$
h_{1}, h_{2} \sim \mathcal{N}\left(\mu_{h}, \frac{\left(1-\rho_{h}\right) \omega_{h}^{2}}{\left(1+\rho_{h}\right)\left(\left(1-\rho_{h}\right)^{2}-\phi_{h}^{2}\right)}\right) .
$$

This stochastic volatility model is referred to as SV-2, which reduces to the standard SV model when $\rho_{h}=0$.

Similar to the GARCH-J model, the third stochastic volatility model accommodates the possibility of infrequent jumps. Specifically, under the stochastic volatility model with jumps (SV-J), the observation equation becomes:

$$
y_{t}=\mu+k_{t} q_{t}+\varepsilon_{t}^{y}, \quad \varepsilon_{t}^{y} \sim \mathcal{N}\left(0, \mathrm{e}^{h_{t}}\right),
$$

where the log-volatility $h_{t}$ follows the same AR(1) process as in (4). The jump indicator $q_{t}$ and jump size $k_{t}$ are modeled exactly the same as in the GARCH-J model.

Next we consider the stochastic volatility in mean (SV-M) model of Koopman and Hol Uspensky (2002), under which the stochastic volatility enters the observation equation as a covariate:

$$
y_{t}=\mu+\lambda \mathrm{e}^{h_{t}}+\varepsilon_{t}^{y}, \quad \varepsilon_{t}^{y} \sim \mathcal{N}\left(0, \mathrm{e}^{h_{t}}\right) .
$$

As before, the log-volatility follows the same $\mathrm{AR}(1)$ process as in (4). The parameter $\lambda$ captures the extent of volatility feedback; when $\lambda=0$, the SV-M reduces to the standard SV model.

The final model is a version of the stochastic volatility models with moving average innovations in Chan (2013). In particular, consider the following first-order moving average model with stochastic volatility:

$$
\begin{aligned}
y_{t} & =\mu+\varepsilon_{t}^{y}, \\
\varepsilon_{t}^{y} & =u_{t}+\psi u_{t-1}, \quad u_{t} \sim \mathcal{N}\left(0, \mathrm{e}^{h_{t}}\right),
\end{aligned}
$$

where $u_{0}=0$ and $|\psi|<1$. Again the log-volatility $h_{t}$ is assumed to follow the $\operatorname{AR}(1)$ process as in (4). This stochastic volatility model is referred to as SV-MA. 
We summarize the GARCH and stochastic volatility models in Table 1. Both the GARCH and stochastic volatility models are estimated using Bayesian techniques. The estimation is outlined in Appendix A.

Table 1: List of GARCH and stochastic volatility models.

\begin{tabular}{ll}
\hline \hline & \multicolumn{1}{c}{ GARCH models } \\
\hline GARCH & GARCH(1,1) model where $\sigma_{t}^{2}$ follows a stationary AR(1) \\
GARCH-2 & same as GARCH but $\sigma_{t}^{2}$ follows a stationary AR $(2)$ \\
GARCH-J & same as GARCH but the prices equation has a "jump" component \\
GARCH-M & same as GARCH but $\sigma_{t}^{2}$ enters the prices equation as a covariate \\
GARCH-MA & same as GARCH but the observation error follows an MA $(1)$ \\
\hline \multicolumn{1}{c}{ Stochastic volatility models } \\
\hline SV & stochastic volatility model where $h_{t}$ follows a stationary AR $(1)$ \\
SV-2 & same as SV but $h_{t}$ follows a stationary AR $(2)$ \\
SV-J & same as SV but the prices equation has a "jump" component \\
SV-M & same as SV but $h_{t}$ enters the prices equation as a covariate \\
SV-MA & same as SV but the observation error follows an MA $(1)$ \\
\hline \hline
\end{tabular}

\section{Model Comparison Using the Bayes Factor}

In this section, we give an overview of Bayesian model comparison via the Bayes factor and outline an efficient approach to compute the Bayes factor using importance sampling.

Suppose we wish to compare a set of models $\left\{M_{1}, \ldots, M_{K}\right\}$. Each model $M_{k}$ is formally defined by two separate components: a likelihood function $p\left(\mathbf{y} \mid \boldsymbol{\theta}_{k}, M_{k}\right)$ that depends on the model-specific parameter vector $\boldsymbol{\theta}_{k}$ and a prior density $p\left(\boldsymbol{\theta}_{k} \mid M_{k}\right)$. One popular Bayesian model comparison criterion is the Bayes factor in favor of $M_{i}$ against $M_{j}$, defined as

$$
\mathrm{BF}_{i j}=\frac{p\left(\mathbf{y} \mid M_{i}\right)}{p\left(\mathbf{y} \mid M_{j}\right)}
$$

where

$$
p\left(\mathbf{y} \mid M_{k}\right)=\int p\left(\mathbf{y} \mid \boldsymbol{\theta}_{k}, M_{k}\right) p\left(\boldsymbol{\theta}_{k} \mid M_{k}\right) \mathrm{d} \boldsymbol{\theta}_{k}
$$

is the marginal likelihood under model $M_{k}, k=i, j$. This marginal likelihood can be interpreted as a density forecast of the data under model $M_{k}$ evaluated at the actual observed data $\mathbf{y}$. Hence, if the observed data are likely under the model, the associated marginal likelihood would be "large". Since the marginal likelihood is essentially a density forecast evaluation, it has a built-in penalty for model complexity. In addition, it follows 
that $\mathrm{BF}_{i j}>1$ indicates that the observed data are more likely under model $M_{i}$ compared to model $M_{j}$, and is thus viewed as evidence in favor of model $M_{i}$ - the weight of evidence is proportional to the value of the Bayes factor.

Furthermore, the Bayes factor is related to the posterior odds ratio between the two models as follows:

$$
\frac{\mathbb{P}\left(M_{i} \mid \mathbf{y}\right)}{\mathbb{P}\left(M_{j} \mid \mathbf{y}\right)}=\frac{\mathbb{P}\left(M_{i}\right)}{\mathbb{P}\left(M_{j}\right)} \times \mathrm{BF}_{i j}
$$

where $\mathbb{P}\left(M_{i}\right) / \mathbb{P}\left(M_{j}\right)$ is the prior odds ratio. If both models are equally probable a priori, i.e., the prior odds ratio is one, the posterior odds ratio between the two models is then equal to the Bayes factor. Then, if, for example, $\mathrm{BF}_{i j}=10$, then model $M_{i}$ is 10 times more likely than model $M_{j}$ given the data. For a more detailed discussion of the Bayes factor, we refer the readers to Koop (2003) and Kroese and Chan (2014).

Since the Bayes factor is simply a ratio of two marginal likelihoods, researchers often only report the marginal likelihoods of the set of competing models. We follow this practice. Next, we outline a method for calculating the marginal likelihoods under the GARCH and stochastic volatility models.

Generally the computation of the marginal likelihood is nontrivial - the integral in (5) is often high-dimensional and cannot be obtained analytically. In this paper we follow Chan and Eisenstat (2015) and use an adaptive importance sampling method known as the cross-entropy method (Rubinstein, 1997; Rubinstein and Kroese, 2004) to compute the marginal likelihood.

The main idea is as follows. There is an ideal importance sampling density that would in principle give a zero-variance importance sampling estimator for the marginal likelihood. However, this density is only known up to a constant and therefore cannot be used as an importance sampling density — which would require the normalizing constant to be known. One way to get around this problem is to instead locate a density within a convenient family of distributions such that its Kullback-Leibler divergence - or the cross-entropy distance - to the ideal density is minimized. Once the optimal density is obtained, it is used to construct the importance sampling estimator.

The main advantage of this adaptive importance sampling method is that it is easy to implement and the numerical standard error of the estimator is readily available. The method only requires the evaluation of the prior and the likelihood. For GARCH models the likelihood can be quickly evaluated. For stochastic volatility models, the completedata likelihood-i.e., the joint distribution of the data and the log-volatilities - can be 
readily evaluated. But the likelihood or more precisely the observed-data likelihood-i.e, the marginal distribution of the data unconditional on the log-volatilities - does not have a closed-form expression. Instead, we use the importance sampling algorithms in Chan and Grant (2014) to evaluate the observed-data likelihood. ${ }^{1}$ We leave the technical details to Appendix B.

\section{Empirical Results}

In this section we compare the performance of the five GARCH models and their stochastic volatility counterparts discussed in Section 2 in fitting weekly price changes on nine series of energy prices. The main goal of this exercise is to examine which class of timevarying volatility models tends to fit the dynamics of a broad range of energy prices better - while controlling for model complexity. In addition, we also seek to establish the type of features that are useful in modeling these prices. For example, does adding an additional channel of volatility feedback fit the data better? Or is it more important to allow for short-run dynamics via a moving average component?

Table 2: Energy price data.

\begin{tabular}{ll}
\hline \hline & \multicolumn{1}{c}{ Crude oil (US Dollars per Barrel) } \\
\hline S1 & Cushing, OK West Texas Intermediate \\
S2 & Europe Brent \\
\hline \multicolumn{2}{c}{ Petroleum products (US Dollars per Gallon) } \\
\hline S3 & NY Harbor Conventional Gasoline Regular \\
S4 & US Gulf Coast Conventional Gasoline Regular \\
S5 & NY Harbor No. 2 Heating Oil \\
S6 & Los Angeles, CA Ultra-Low Sulfur CARB Diesel \\
S7 & US Gulf Coast Kerosene-Type Jet Fuel \\
S8 & Mont Belvieu, TX Propane \\
\hline & Natural gas (US Dollars per Million Btu) \\
\hline S9 & Henry Hub Natural Gas \\
\hline \hline
\end{tabular}

With these aims in mind, we choose a broad range of energy prices that are commonly

\footnotetext{
${ }^{1}$ It is also worth noting that the method of Gelfand and Dey (1994) is often used in conjunction with the complete-data likelihood to compute the marginal likelihood. However, using an empirical example, Chan and Grant (2015) show that this approach can have a substantial finite-sample bias in the marginal likelihood estimate.
} 
used in empirical applications. More specifically, we obtain the nine series of (FOB) spot prices of crude oil, various petroleum products and natural gas from the US Energy Information Administration. The specific details of each data series are contained in Table 2. The data frequency is weekly and the sample period is from 3 January 1997 to 6 February 2015. The data are transformed into nominal rates of change by taking the first difference of the logs and multiplying by 100 .

\subsection{Model Comparison Results}

All the models are estimated using the Bayesian techniques outlined in Appendix A. The marginal likelihoods are computed using the cross-entropy method of Chan and Eisenstat (2015), which is discussed in Appendix B. The results are reported in Table 3.

Table 3: Log marginal likelihoods of the GARCH and SV models for the nine series of weekly price changes on energy prices. The numerical standard errors are in parentheses.

\begin{tabular}{lccccccccc}
\hline \hline & $\mathrm{S} 1$ & $\mathrm{~S} 2$ & $\mathrm{~S} 3$ & $\mathrm{~S} 4$ & $\mathrm{~S} 5$ & $\mathrm{~S} 6$ & $\mathrm{~S} 7$ & $\mathrm{~S} 8$ & $\mathrm{~S} 9$ \\
\hline GARCH & -2647.0 & -2652.0 & -2809.7 & -2840.0 & -2647.0 & -2718.3 & -2634.5 & -2679.2 & -3117.0 \\
& $(0.05)$ & $(0.02)$ & $(0.02)$ & $(0.02)$ & $(0.02)$ & $(0.02)$ & $(0.02)$ & $(0.01)$ & $(0.10)$ \\
SV & -2632.4 & -2636.7 & -2783.8 & -2822.6 & -2612.2 & -2693.5 & -2623.1 & -2644.0 & -3058.6 \\
& $(0.01)$ & $(0.01)$ & $(0.01)$ & $(0.01)$ & $(0.01)$ & $(0.02)$ & $(0.01)$ & $(0.06)$ & $(0.04)$ \\
GARCH-2 & -2647.2 & -2652.2 & -2807.6 & -2839.4 & -2647.3 & -2712.1 & -2634.4 & -2679.7 & -3118.1 \\
& $(0.10)$ & $(0.09)$ & $(0.04)$ & $(0.05)$ & $(0.11)$ & $(0.04)$ & $(0.06)$ & $(0.04)$ & $(0.04)$ \\
SV-2 & -2631.9 & -2636.4 & -2783.5 & -2821.9 & -2611.8 & -2692.2 & -2622.6 & -2641.2 & -3057.9 \\
& $(0.08)$ & $(0.07)$ & $(0.06)$ & $(0.11)$ & $(0.06)$ & $(0.10)$ & $(0.10)$ & $(0.08)$ & $(0.06)$ \\
GARCH-J & -2634.6 & -2638.4 & -2789.7 & -2822.1 & -2613.0 & -2700.1 & -2627.7 & -2650.3 & -3061.0 \\
& $(0.03)$ & $(0.07)$ & $(0.05)$ & $(0.05)$ & $(0.11)$ & $(0.06)$ & $(0.03)$ & $(0.06)$ & $(0.06)$ \\
SV-J & -2632.3 & -2637.1 & -2784.4 & -2823.1 & -2613.4 & -2694.3 & -2624.0 & -2645.1 & -3059.2 \\
& $(0.04)$ & $(0.02)$ & $(0.02)$ & $(0.03)$ & $(0.03)$ & $(0.03)$ & $(0.02)$ & $(0.06)$ & $(0.04)$ \\
GARCH-M & -2653.6 & -2658.8 & -2817.0 & -2847.3 & -2654.0 & -2725.4 & -2641.3 & -2680.6 & -3123.6 \\
& $(0.02)$ & $(0.03)$ & $(0.04)$ & $(0.02)$ & $(0.03)$ & $(0.02)$ & $(0.02)$ & $(0.03)$ & $(0.04)$ \\
SV-M & -2637.8 & -2642.9 & -2790.3 & -2828.8 & -2618.8 & -2699.9 & -2629.1 & -2646.2 & -3065.3 \\
& $(0.02)$ & $(0.02)$ & $(0.13)$ & $(0.04)$ & $(0.02)$ & $(0.04)$ & $(0.03)$ & $(0.04)$ & $(0.03)$ \\
GARCH-MA & -2630.7 & -2620.4 & -2798.6 & -2829.5 & -2621.8 & -2673.5 & -2616.3 & -2653.6 & -3107.7 \\
& $(0.03)$ & $(0.04)$ & $(0.02)$ & $(0.02)$ & $(0.16)$ & $(0.07)$ & $(0.04)$ & $(0.02)$ & $(0.04)$ \\
SV-MA & -2615.9 & -2610.1 & -2771.8 & -2806.6 & -2588.6 & -2648.6 & -2606.6 & -2622.1 & -3048.7 \\
& $(0.01)$ & $(0.01)$ & $(0.01)$ & $(0.02)$ & $(0.01)$ & $(0.02)$ & $(0.01)$ & $(0.05)$ & $(0.02)$ \\
\hline \hline
\end{tabular}

A few broad conclusions can be drawn from this exercise. Overall, the best model is the SV-MA for all the nine time series. The second place is less clear-cut; the SV-2 often 
comes out on top, although the GARCH-MA does better for four series. Second, with the notable exception of the GARCH-J and SV-J pair, the SV models always outperform their GARCH counterparts. As an example, consider the results for S1 (Cushing, OK West Texas Intermediate). The log marginal likelihoods of the GARCH and SV models are, respectively, -2647.0 and -2632.4. This implies a Bayes factor of $2.2 \times 10^{6}$ in favor of the SV model against its GARCH counterpart, indicating overwhelming evidence for the former model. For the same series, the Bayes factor in favor of the SV-MA against the GARCH-MA is $2.7 \times 10^{6}$, again showing overwhelming evidence in support of the former model.

The exception of this general pattern is the GARCH-J and SV-J pair, where the GARCH$\mathrm{J}$ performs slightly better than the SV-J for two out of the nine series. As mentioned earlier, under GARCH models the conditional variance is a deterministic function of the parameters and past data - in contrast to stochastic volatility models, in which the logvolatility is a random variable. As such, stochastic volatility models are more robust to misspecification and to drastic changes in the time series. This helps explain why they tend to outperform their GARCH counterparts. However, when a jump componentwhich is a random variable - is added to a GARCH, it gives the model extra flexibility against misspecification and outliers, making the inherent advantage of SV models less apparent. This also explains why the GARCH-J does substantially better than the GARCH in the model comparison exercise, ${ }^{2}$ whereas the SV-J and SV give very similar resultse.g., in the SV a "jump" can be partially accommodated by a large shock in the stochastic volatility process.

Now, we further investigate which features are important in modeling the dynamics of energy prices. By comparing the GARCH with GARCH-2 and the SV with SV-2, we conclude that the richer $\mathrm{AR}(2)$ volatility process provides only marginal benefits. For example, the Bayes factor in favor of the SV-2 against the SV is only 2 for S4 (US Gulf Coast Conventional Gasoline Regular). Results for the other series are broadly similar. Thus, for modeling energy prices at least, one can feel comfortable maintaining the conventional specification of an $\mathrm{AR}(1)$ volatility process.

Next, we examine the importance of volatility feedback for modeling energy prices. Although this channel is found to be empirically important for stock returns, it is superfluous for energy data. In fact, adding the volatility feedback component often markedly de-

\footnotetext{
${ }^{2}$ Mason and Wilmot (2014) also find that allowing for a jump process substantially improves the fit of the GARCH model for natural gas spot prices.
} 
creases the marginal likelihood of a model. For instance, the Bayes factor in favor of the GARCH against the GARCH-M is about 1200 for S6 (Los Angeles, CA Ultra-Low Sulfur CARB Diesel). This is in line with the finding in Sadorsky (2006), who finds that GARCH in mean forecasts no better than the standard GARCH for crude oil, heating oil and natural gas volatility. It is also worth noting that even though the GARCH-M nests GARCH as a special case - hence, the GARCH-M would fit the data better-the Bayes factor still prefers the simpler model, highlighting its built-in penalty against model complexity.

To investigate the relevance of the MA component, we compare the GARCH with GARCHMA and the SV with SV-MA. For both classes of models, adding the MA component drastically improves the model-fit for all series. For example, the Bayes factor in favor of the SV-MA against the SV is $3.2 \times 10^{9}$ for S8 (Mont Belvieu, TX Propane), indicating that the weekly returns exhibit substantial serial correlation. Similar results are obtained for the GARCH models and for the other price series.

\subsection{Estimation Results}

In this section we report the posterior estimates of the model parameters for both the GARCH and stochastic volatility models. Due to space constraint, we only present results for the crude oil price (Cushing, OK West Texas Intermediate), which are broadly representative of the estimates for other energy prices.

Table 4 shows the results for the GARCH models. The parameters governing the evolution of the conditional variance process have similar estimates across models. In particular, all indicate high persistence with the posterior mean of $\beta_{1}$ estimated to be between 0.74 to 0.9 - the lowest estimate is from the GARCH-2, in which the sum of $\beta_{1}$ and $\beta_{2}$ is estimated to be 0.88 , implying a persistence level similar to other models. The estimate of $\beta_{2}$ is small and an $\mathrm{AR}(1)$ for the conditional variance process appears to be sufficient, which supports the ranking of the marginal likelihood.

The average jump size $\mu_{k}$ is estimated to be negative at about $-1.4 \%$. The estimate for the jump probability $\kappa$ is 0.05 , which implies about 2.5 "jumps" per year for weekly data. It is interesting to note that the posterior estimates of $\lambda$ and $\psi$ both seem to support the ranking of the marginal likelihood. For example, recall that when $\psi=0$, the GARCH-MA reduces to the standard GARCH. Since the marginal likelihood favors the GARCH-MA relative to the GARCH, one would expect that the posterior distribution 
of $\psi$ has little mass around zero. In fact, the $95 \%$ credible interval of $\psi$ is estimated to be $(0.16,0.31)$, which excludes 0 . Similarly, when $\lambda=0$, the GARCH-M reduces to the standard GARCH. The $95 \%$ credible interval of $\lambda$ is estimated to be $(-0.01,0.04)$, which includes 0 , supporting the ranking of the marginal likelihood that favors the GARCH over the GARCM-M.

Table 4: Parameter posterior means and standard deviations (in parentheses) for the GARCH models (S1: Cushing, OK West Texas Intermediate).

\begin{tabular}{cccccc}
\hline \hline & GARCH & GARCH-2 & GARCH-J & GARCH-M & GARCH-MA \\
\hline$\mu$ & 0.15 & 0.15 & 0.20 & -0.10 & 0.16 \\
& $(0.12)$ & $(0.12)$ & $(0.13)$ & $(0.23)$ & $(0.14)$ \\
$\alpha_{0}$ & 0.65 & 0.51 & 0.41 & 0.48 & 0.37 \\
& $(0.22)$ & $(0.18)$ & $(0.16)$ & $(0.17)$ & $(0.14)$ \\
$\alpha_{1}$ & 0.10 & 0.10 & 0.08 & 0.08 & 0.08 \\
& $(0.02)$ & $(0.02)$ & $(0.02)$ & $(0.01)$ & $(0.01)$ \\
$\beta_{1}$ & 0.87 & 0.74 & 0.88 & 0.89 & 0.90 \\
& $(0.03)$ & $(0.04)$ & $(0.02)$ & $(0.02)$ & $(0.01)$ \\
$\beta_{2}$ & - & 0.14 & - & - & - \\
& - & $(0.03)$ & - & - & - \\
$\kappa$ & - & - & 0.05 & - & - \\
& - & - & $(0.02)$ & - & - \\
$\mu_{k}$ & - & - & -1.39 & - & - \\
& - & - & $(0.93)$ & - & - \\
$\sigma_{k}^{2}$ & - & - & 41.26 & - & - \\
& - & - & $(20.82)$ & - & - \\
$\lambda$ & - & - & - & 0.02 & - \\
& - & - & - & $(0.01)$ & - \\
$\psi$ & - & - & - & - & 0.24 \\
& - & - & - & - & $(0.04)$ \\
\hline \hline
\end{tabular}

Next, we present the parameter estimates for the stochastic volatility models in Table 5. Similar to the estimates under GARCH models, the stochastic volatility process is highly persistent for all models. In particular, the posterior mean of $\phi_{h}$ is estimated to be between 0.96 to 0.97 across the various models. Other parameters governing the stochastic volatility process are also similar across models.

In contrast to the GARCH-J results, the average jump size $\mu_{k}$ under SV-J is estimated to be positive (but with a large posterior standard deviation). Seemingly very different "jumps" are identified under the SV-J compared to the GARCH-J. Given the small estimate for $\mu_{k}$, the marginal likelihood favors the SV model compared to SV-J, whereas 
among the GARCH and GARCH-J pair it prefers the latter model.

The estimate of the moving average parameter is similar across the GARCH- MA and SV-MA models, with $\psi$ estimated to be 0.22 under the SV-MA. Its $95 \%$ credible interval is $(0.15,0.29)$, which excludes 0 . Hence, these estimation results also support the ranking of the marginal likelihood which favors the SV-MA against the standard SV. Lastly, the coefficient of the volatility $\lambda$ is estimated to be -0.02 with a $95 \%$ credible interval $(-0.05$, 0.01), indicating that volatility feedback is unimportant for modeling crude oil returns.

Table 5: Parameter posterior means and standard deviations (in parentheses) for the stochastic volatility models (S1: Cushing, OK West Texas Intermediate).

\begin{tabular}{cccccc}
\hline \hline & SV & SV-2 & SV-J & SV-M & SV-MA \\
\hline$\mu$ & 0.17 & 0.19 & 0.18 & 0.47 & 0.16 \\
& $(0.12)$ & $(0.12)$ & $(0.14)$ & $(0.24)$ & $(0.14)$ \\
$\mu_{h}$ & 2.67 & 2.66 & 2.63 & 2.67 & 2.63 \\
& $(0.22)$ & $(0.25)$ & $(0.23)$ & $(0.22)$ & $(0.25)$ \\
$\phi_{h}$ & 0.97 & 0.96 & 0.97 & 0.97 & 0.97 \\
& $(0.01)$ & $(0.08)$ & $(0.01)$ & $(0.01)$ & $(0.01)$ \\
$\omega_{h}^{2}$ & 0.03 & 0.05 & 0.03 & 0.03 & 0.03 \\
& $(0.01)$ & $(0.01)$ & $(0.01)$ & $(0.01)$ & $(0.01)$ \\
$\rho_{h}$ & - & -0.03 & - & - & - \\
& - & $(0.09)$ & - & - & - \\
$\kappa$ & - & - & 0.04 & - & - \\
& - & - & $(0.03)$ & - & - \\
$\mu_{k}$ & - & - & 0.22 & - & - \\
& - & - & $(1.67)$ & - & - \\
$\sigma_{k}^{2}$ & - & - & 18.56 & - & - \\
& - & - & $(22.06)$ & - & - \\
$\lambda$ & - & - & - & -0.02 & - \\
& - & - & - & $(0.02)$ & - \\
$\psi$ & - & - & - & - & 0.22 \\
& - & - & - & - & $(0.03)$ \\
\hline \hline
\end{tabular}

\section{Concluding Remarks and Future Research}

We have undertaken a formal Bayesian model comparison exercise to assess a number of GARCH and stochastic volatility models for modeling oil, petroleum product and natural gas prices. Using the marginal likelihood to assess the various models, we find that stochastic volatility models almost always outperform their GARCH counterparts, 
suggesting that stochastic volatility models might provide a better alternative to the more conventional GARCH models. Overall, the stochastic volatility model with moving average innovations is the best model for all nine series considered.

For future research, it would be worthwhile to compare multivariate GARCH and stochastic volatility models in fitting multiple energy prices. In particular, it would be important to determine the number of sources of fluctuation in these series. Moreover, it would also be interesting to incorporate macroeconomic variables in the multivariate analysis, as the interplay between energy prices and macroeconomic variables is often of interest.

\section{Appendix A: Priors and Estimation}

In this appendix we discuss the priors and outline the estimation methods for fitting the GARCH and stochastic volatility models discussed in Section 2.

\section{Priors}

We choose broadly similar priors across the GARCH and stochastic volatility models. In particular, we use the same prior for common parameters. All priors are proper but relatively noninformative.

For the standard GARCH, we assume the following independent priors for $\mu$ and $\gamma=$ $\left(\alpha_{0}, \alpha_{1}, \beta_{1}\right)^{\prime}$ :

$$
\mu \sim \mathcal{N}\left(\mu_{0}, V_{\mu}\right), \quad \log \gamma \sim \mathcal{N}\left(\gamma_{0}, \mathbf{V}_{\gamma}\right) \mathbb{1}\left(\alpha_{1}+\beta_{1}<1\right)
$$

that is, $\gamma$ follows a truncated log-normal distribution with the stationarity restriction that $\alpha_{1}+\beta_{1}<1$. We set the hyperparameters to be $\mu_{0}=0, V_{\mu}=10, \gamma_{0}=(1, \log 0.1, \log 0.8)^{\prime}$ and $\mathbf{V}_{\boldsymbol{\gamma}}=\operatorname{diag}(10,1,1)$. These values imply relatively noninformative priors with prior medians that are similar to typical estimates from financial data. In particular, the prior medians of $\mu$ and $\gamma$ are respectively 0 and $(2.72,0.1,0.8)^{\prime}$. For the GARCH2, we use the same prior for $\mu$, but replace the prior for $\gamma$ with a prior for $\widetilde{\gamma}=\left(\alpha_{0}, \alpha_{1}, \beta_{1}, \beta_{2}\right)^{\prime}$ where $\log \widetilde{\gamma} \sim \mathcal{N}\left(\widetilde{\gamma}_{0}, \mathbf{V}_{\widetilde{\gamma}}\right) \mathbb{1}\left(\alpha_{1}+\beta_{1}+\beta_{2}<1\right)$ with $\widetilde{\gamma}_{0}=(1, \log 0.1, \log 0.8, \log 0.1)^{\prime}$ and $\mathbf{V}_{\widetilde{\gamma}}=\operatorname{diag}(10,1,1,1)$.

For each of the remaining GARCH models, the priors for $\mu$ and $\gamma$ are exactly the same as in (6). Moreover, under the GARCH-J, the jump intensity $\kappa$ is assumed to have a uniform 
distribution on the interval $(0,0.1)$, and the average jump size and the jump variance $\boldsymbol{\delta}=\left(\mu_{k}, \log \sigma_{k}^{2}\right)^{\prime}$ are distributed as a bivariate normal distribution: $\kappa \sim \mathcal{U}(0,0.1)$ and $\boldsymbol{\delta} \sim \mathcal{N}\left(\boldsymbol{\delta}_{0}, \mathbf{V}_{\boldsymbol{\delta}}\right)$. We set $\boldsymbol{\delta}_{0}=(0, \log 10)^{\prime}$ and $\mathbf{V}_{\boldsymbol{\delta}}=\operatorname{diag}(10,1)$ so that the average jump size is 0 . For the GARCH-M, the coefficient of the volatility is assumed to have a normal distribution: $\lambda \sim \mathcal{N}\left(\lambda_{0}, V_{\lambda}\right)$, where $\lambda_{0}=0$ and $V_{\lambda}=100$. Finally, the MA(1) coefficient in the GARCH-MA has a normal distribution truncated within the unit interval: $\psi \sim$ $\mathcal{N}\left(\psi_{0}, V_{\psi}\right) \mathbb{1}(|\psi|<1)$, where $\psi_{0}=0$ and $V_{\psi}=1$.

Next, we discuss the set of priors for each of the stochastic volatility models. In general, we choose the same hyperparameters for parameters that are common across models. For the standard SV, we assume the following independent priors for $\mu, \mu_{h}, \phi_{h}$ and $\omega_{h}^{2}$ :

$$
\begin{array}{rlrl}
\mu & \sim \mathcal{N}\left(\mu_{0}, V_{\mu}\right), & \mu_{h} & \sim \mathcal{N}\left(\mu_{h 0}, V_{\mu_{h}}\right), \\
\phi_{h} & \sim \mathcal{N}\left(\phi_{h 0}, V_{\phi_{h}}\right) \mathbb{1}\left(\left|\phi_{h}\right|<1\right), & \omega_{h}^{2} \sim \mathcal{I} \mathcal{G}\left(\nu_{h}, S_{h}\right),
\end{array}
$$

where $\mathcal{I} \mathcal{G}(\cdot, \cdot)$ denotes the inverse-gamma distribution. We set $\mu_{0}=0, \mu_{h 0}=1, V_{\mu}=$ $V_{\mu_{h}}=10, \phi_{h 0}=0.97, V_{\phi_{h}}=0.1^{2}, \nu_{h}=5$ and $S_{h}=0.16$. These hyperparameters are set so that the stochastic volatility process has similar dynamics as the conditional variance under the GARCH models.

For the SV2, we assume the same priors for $\mu, \mu_{h}$ and $\omega_{h}^{2}$ as in (7), but replace the prior for $\phi_{h}$ with a prior for $\boldsymbol{\theta}_{h}=\left(\phi_{h}, \rho_{h}\right)^{\prime}: \boldsymbol{\theta}_{h} \sim \mathcal{N}\left(\boldsymbol{\theta}_{h 0}, \mathbf{V}_{\boldsymbol{\theta}_{h}}\right) \mathbb{1}\left(\boldsymbol{\theta}_{h} \in \mathbf{A}\right)$, where $\boldsymbol{\theta}_{h 0}=(0.97,0)^{\prime}$, $\mathbf{V}_{\boldsymbol{\theta}_{h}}=\operatorname{diag}\left(0.1^{2}, 1\right)$ and $\mathbf{A} \subset \mathbb{R}^{2}$ is the set where the roots of the characteristic polynomial defined by $\boldsymbol{\theta}_{h}$ lie outside the unit circle. For each of the remaining stochastic volatility models, the priors for $\mu, \mu_{h}, \phi_{h}$ and $\omega_{h}^{2}$ are the same as in (7). The additional parameters have exactly the same priors as their GARCH counterparts.

\section{Bayesian Estimation}

All the GARCH and stochastic volatility models are estimated using Markov chain Monte Carlo (MCMC) methods. Specifically, we sample from the posterior distributions of the models by constructing Markov samplers and use the posterior draws obtained to compute various quantities of interest such as the posterior means and the marginal likelihoods.

For the stochastic volatility models, a key step is to jointly sample the log-volatilities. For example, under the standard SV model, we need to sample from the conditional density $p\left(\mathbf{h} \mid \mathbf{y}, \mu, \mu_{h}, \phi_{h}, \omega_{h}^{2}\right)$. This is done using the accept-reject Metropolis-Hastings algorithm 
described in Chan (2014), which is based on the precision sampler of Chan and Jeliazkov (2009). A key feature of this algorithm is its use of fast band matrix routines rather than using the conventional Kalman filter. The former approach is in general more efficient than the latter.

To implement the accept-reject Metropolis-Hastings algorithm, one key ingredient is an appropriate proposal density that well approximates the target $p\left(\mathbf{h} \mid \mathbf{y}, \mu, \mu_{h}, \phi_{h}, \omega_{h}^{2}\right)$. The basic idea is to approximate the target using a Gaussian density. To that end, note that $p\left(\mathbf{h} \mid \mathbf{y}, \mu, \mu_{h}, \phi_{h}, \omega_{h}^{2}\right) \propto p(\mathbf{y} \mid \mu, \mathbf{h}) p\left(\mathbf{h} \mid \mu_{h}, \phi_{h}, \omega_{h}^{2}\right)$. It can be shown that the latter density $p\left(\mathbf{h} \mid \mu_{h}, \phi_{h}, \omega_{h}^{2}\right)$ is Gaussian. In fact, we have

$$
\log p\left(\mathbf{h} \mid \mu_{h}, \phi_{h}, \omega_{h}^{2}\right)=-\frac{1}{2}\left(\mathbf{h}^{\prime} \mathbf{H}_{\phi_{h}}^{\prime} \boldsymbol{\Sigma}_{h}^{-1} \mathbf{H}_{\phi_{h}} \mathbf{h}-2 \mathbf{h}^{\prime} \mathbf{H}_{\phi_{h}}^{\prime} \boldsymbol{\Sigma}_{h}^{-1} \mathbf{H}_{\phi_{h}} \boldsymbol{\delta}_{h}\right)+c_{1},
$$

where $c_{1}$ is a constant independent of $\mathbf{h}$,

$$
\mathbf{H}_{\phi_{h}}=\left(\begin{array}{ccccc}
1 & 0 & 0 & \cdots & 0 \\
-\phi_{h} & 1 & 0 & \ldots & 0 \\
0 & -\phi_{h} & 1 & \cdots & 0 \\
\vdots & \ddots & \ddots & \ddots & \vdots \\
0 & 0 & \cdots & -\phi_{h} & 1
\end{array}\right)
$$

is a lower triangular matrix, $\boldsymbol{\Sigma}_{h}=\operatorname{diag}\left(\omega_{h}^{2} /\left(1-\phi_{h}^{2}\right), \omega_{h}^{2}, \ldots, \omega_{h}^{2}\right)$ and $\boldsymbol{\delta}_{h}=\mathbf{H}_{\phi_{h}}^{-1} \widetilde{\boldsymbol{\delta}}_{h}$ with $\widetilde{\boldsymbol{\delta}}_{h}=\left(\mu_{h},\left(1-\phi_{h}\right) \mu_{h}, \ldots,\left(1-\phi_{h}\right) \mu_{h}\right)^{\prime}$.

Next, we approximate $p(\mathbf{y} \mid \mu, \mathbf{h})$ by a Gaussian density in $\mathbf{h}$. To that end, we expand $\log p(\mathbf{y} \mid \mu, \mathbf{h})=\sum_{t=1}^{T} \log p\left(y_{t} \mid \mu, h_{t}\right)$ around the point $\widetilde{\mathbf{h}}$ - which is chosen to be the mode of $p\left(\mathbf{h} \mid \mathbf{y}, \mu, \mu_{h}, \phi_{h}, \omega_{h}^{2}\right)$-by a second-order Taylor expansion:

$$
\begin{aligned}
\log p(\mathbf{y} \mid \mu, \mathbf{h}) & \approx \log p(\mathbf{y} \mid \mu, \widetilde{\mathbf{h}})+(\mathbf{h}-\widetilde{\mathbf{h}})^{\prime} \mathbf{f}-\frac{1}{2}(\mathbf{h}-\widetilde{\mathbf{h}})^{\prime} \mathbf{G}(\mathbf{h}-\widetilde{\mathbf{h}}) \\
& =-\frac{1}{2}\left(\mathbf{h}^{\prime} \mathbf{G} \mathbf{h}-2 \mathbf{h}^{\prime}(\mathbf{f}+\mathbf{G} \widetilde{\mathbf{h}})\right)+c_{2}
\end{aligned}
$$

where $c_{2}$ is a constant independent of $\mathbf{h}, \mathbf{f}=\left(f_{1}, \ldots, f_{T}\right)^{\prime}$ and $\mathbf{G}=\operatorname{diag}\left(G_{1}, \ldots, G_{T}\right)$ with

$$
f_{t}=\left.\frac{\partial}{\partial h_{t}} \log p\left(y_{t} \mid \mu, h_{t}\right)\right|_{h_{t}=\widetilde{h}_{t}}, \quad G_{t}=-\left.\frac{\partial^{2}}{\partial h_{t}^{2}} \log p\left(y_{t} \mid \mu, h_{t}\right)\right|_{h_{t}=\widetilde{h}_{t}} .
$$

That is, $\mathbf{G}$ is the negative Hessian of the log-density evaluated at $\widetilde{\mathbf{h}}$. For the standard stochastic volatility model, $\mathbf{G}$ is diagonal (hence a band matrix). 
Finally, combining (8) and (9), we have

$$
\begin{aligned}
\log p\left(\mathbf{h} \mid \mathbf{y}, \mu, \mu_{h}, \phi_{h}, \omega_{h}^{2}\right) & =\log p(\mathbf{y} \mid \mu, \mathbf{h})+\log p\left(\mathbf{h} \mid \mu_{h}, \phi_{h}, \omega_{h}^{2}\right)+c_{3}, \\
& \approx-\frac{1}{2}\left(\mathbf{h}^{\prime} \mathbf{K}_{\mathbf{h}} \mathbf{h}-2 \mathbf{h}^{\prime} \mathbf{k}_{\mathbf{h}}\right)+c_{4},
\end{aligned}
$$

where $c_{3}$ and $c_{4}$ are constants independent of $\mathbf{h}, \mathbf{K}_{\mathbf{h}}=\mathbf{H}_{\phi_{h}}^{\prime} \boldsymbol{\Sigma}_{h}^{-1} \mathbf{H}_{\phi_{h}}+\mathbf{G}$ and $\mathbf{k}_{\mathbf{h}}=$ $\mathbf{f}+\mathbf{G} \widetilde{\mathbf{h}}+\mathbf{H}_{\phi_{h}}^{\prime} \boldsymbol{\Sigma}_{h}^{-1} \mathbf{H}_{\phi_{h}} \boldsymbol{\delta}_{\mathbf{h}}$. It can be shown that the expression in (10) is the log-kernel of the $\mathcal{N}\left(\widehat{\mathbf{h}}, \mathbf{K}_{\mathbf{h}}^{-1}\right)$ density (see, e.g., Kroese and Chan, 2014, p. 238), where $\widehat{\mathbf{h}}=\mathbf{K}_{\mathbf{h}}^{-1} \mathbf{k}_{\mathbf{h}}$. This Gaussian density with mean vector $\widehat{\mathbf{h}}$ and precision matrix $\mathbf{K}_{\mathbf{h}}$ is then used as the proposal density in the accept-reject Metropolis-Hastings algorithm. It is important to note that $\mathbf{K}_{\mathbf{h}}$ is a band matrix, and consequently sampling from this proposal density is fast; see, e.g., Chan and Jeliazkov (2009). We refer the readers to Chan and Grant (2014) for the estimation details of the stochastic volatility models.

For the GARCH models, we use Metropolis-Hastings algorithms to sample from the posterior distributions. For example, for the standard GARCH, we group the parameters into two blocks - $\mu$ and $\gamma=\left(\alpha_{0}, \alpha_{1}, \beta_{1}\right)^{\prime}$-and we draw from the two full conditional distributions $p(\mu \mid \mathbf{y}, \boldsymbol{\gamma})$ and $p(\boldsymbol{\gamma} \mid \mathbf{y}, \mu)$ sequentially. Since both the conditional distributions are nonstandard, ${ }^{3}$ Metropolis-Hastings algorithms are required. To sample $\mu$, we use a Gaussian proposal with mean $\bar{y}$ and variance $s^{2} / T$, where $\bar{y}$ and $s^{2}$ are the sample mean and sample variance respectively. For $\gamma$, we use a Gaussian proposal centered at the mode of $p(\gamma \mid \mathbf{y}, \mu)$ with covariance matrix set to be the outer product of the scores. For other GARCH models with additional parameters, the basic sampler remains the same but with an extra block to sample the additional parameters.

\section{Appendix B: Marginal Likelihood Computation}

The marginal likelihoods for the GARCH and stochastic volatility models are computed using the adaptive importance sampling approach in Chan and Eisenstat (2015). More specifically, the marginal likelihood $p(\mathbf{y})$ for a given model is estimated using:

$$
\widehat{p(\mathbf{y})}=\frac{1}{R} \sum_{i=1}^{R} \frac{p\left(\mathbf{y} \mid \boldsymbol{\theta}^{(i)}\right) p\left(\boldsymbol{\theta}^{(i)}\right)}{g\left(\boldsymbol{\theta}^{(i)}\right)}
$$

\footnotetext{
${ }^{3}$ The conditional distribution of $\mu$ is not Gaussian as $\mu$ also appears in the conditional variance $\sigma_{t}^{2}$.
} 
where $\boldsymbol{\theta}^{(1)}, \ldots, \boldsymbol{\theta}^{(R)}$ are independent draws obtained from the importance density $g(\cdot)$ that dominates the product of the likelihood and the prior $p(\mathbf{y} \mid \cdot) p(\cdot)$. The importance sampling estimator (11) is an unbiased, simulation-consistent estimator of the marginal likelihood $p(\mathbf{y})$. The choice of the importance density is critical for the performance of this estimator. As outlined in Chan and Eisenstat (2015), the theoretical zero-variance importance density for estimating $p(\mathbf{y})$ is the posterior density $p(\boldsymbol{\theta} \mid \mathbf{y})$-which cannot be used as its normalizing constant is unknown. The cross-entropy method generates a procedure to construct an optimal importance density by minimizing the Kullback-Leibler divergence to the zero-variance importance density.

The optimal importance density is typically located within the same parametric class as the prior densities. For example, in the case of the standard GARCH in (1)-(2), the optimal importance density has the form: $g(\boldsymbol{\theta})=p(\mu) p(\boldsymbol{\gamma})$, where the prior densities are given in Appendix A. Once the optimal importance density $g(\boldsymbol{\theta})$ is constructed, the importance sampling estimator in (11) can be obtained easily for the GARCH models, as the (observed-data) likelihood $p(\mathbf{y} \mid \boldsymbol{\theta})$ can be evaluated quickly. For instance, the log-likelihood for the standard GARCH is given by

$$
\log p(\mathbf{y} \mid \mu, \gamma)=-\frac{T}{2} \log (2 \pi)-\frac{1}{2} \sum_{t=1}^{T} \log \sigma_{t}^{2}-\frac{1}{2} \sum_{t=1}^{T} \frac{\left(y_{t}-\mu\right)^{2}}{\sigma_{t}^{2}}
$$

where the conditional variance process $\sigma_{t}^{2}$ is given in (2).

For the stochastic volatility models, the observed-data likelihood $p(\mathbf{y} \mid \boldsymbol{\theta})$ is not available analytically and again we evaluate it using importance sampling. Recall that the observed-data likelihood is given by

$$
p(\mathbf{y} \mid \boldsymbol{\theta})=\int p(\mathbf{y} \mid \boldsymbol{\theta}, \mathbf{h}) p(\mathbf{h} \mid \boldsymbol{\theta}) \mathrm{d} \mathbf{h},
$$

where $p(\mathbf{y} \mid \boldsymbol{\theta}, \mathbf{h})$ is the conditional likelihood and $p(\mathbf{h} \mid \boldsymbol{\theta})$ is the prior density of the logvolatilities $\mathbf{h}$. If $\mathbf{h}^{(1)}, \ldots, \mathbf{h}^{(R)}$ are independent samples from the importance density $g(\mathbf{h})$, then we can estimate the observed-data likelihood $p(\mathbf{y} \mid \boldsymbol{\theta})$ using the following importance sampling estimator:

$$
\widehat{p(\mathbf{y} \mid \boldsymbol{\theta})}=\frac{1}{R} \sum_{i=1}^{R} \frac{p\left(\mathbf{y} \mid \boldsymbol{\theta}, \mathbf{h}^{(i)}\right) p\left(\mathbf{h}^{(i)} \mid \boldsymbol{\theta}\right)}{g\left(\mathbf{h}^{(i)}\right)} .
$$

To choose a suitable $g(\cdot)$, we note that the theoretical zero-variance importance density for estimating $p(\mathbf{y} \mid \boldsymbol{\theta})$ is the conditional density $p(\mathbf{h} \mid \mathbf{y}, \boldsymbol{\theta}) \propto p(\mathbf{y} \mid \boldsymbol{\theta}, \mathbf{h}) p(\mathbf{h} \mid \boldsymbol{\theta})$. Hence, 
we would like to choose $g(\cdot)$ to be "close" to $p(\mathbf{h} \mid \mathbf{y}, \boldsymbol{\theta})$.

Recall that when we estimate the stochastic volatility models, one key step is to approximate the conditional distribution $p(\mathbf{h} \mid \mathbf{y}, \boldsymbol{\theta})$ using a Gaussian density (see Appendix A for details). For example, in the case of the standard SV model, we can use the Gaussian density in (10) as our importance sampling density. For each of the other stochastic volatility models, we can use a similar Gaussian approximation; see Chan and Grant (2014) for details.

\section{References}

P. Agnolucci. Volatility in crude oil futures: A comparison of the predictive ability of GARCH and implied volatility models. Energy Economics, 31(2):316-321, 2009.

O. J. Blanchard and M. Riggi. Why are the 2000s so different from the 1970s? A structural interpretation of changes in the macroeconomic effects of oil prices. Journal of the European Economic Association, 11(5):1032-1052, 2013.

T. Bollerslev. Generalized autoregressive conditional heteroskedasticity. Journal of Econometrics, 31(3):307-327, 1986.

C. Brooks and M. Prokopczuk. The dynamics of commodity prices. Quantitative Finance, 13(4):527-542, 2013.

J. C. C. Chan. Moving average stochastic volatility models with application to inflation forecast. Journal of Econometrics, 176(2):162-172, 2013.

J. C. C. Chan. The stochastic volatility in mean model with time-varying parameters: An application to inflation modeling. CAMA Working Paper, 2014.

J. C. C. Chan and E. Eisenstat. Marginal likelihood estimation with the Cross-Entropy method. Econometric Reviews, 34(3):256-285, 2015.

J. C. C. Chan and A. L. Grant. Issues in comparing stochastic volatility models using the deviance information criterion. CAMA Working Paper, 2014.

J. C. C. Chan and A. L. Grant. Pitfalls of estimating the marginal likelihood using the modified harmonic mean. Economics Letters, 131:29-33, 2015.

J. C. C. Chan and I. Jeliazkov. Efficient simulation and integrated likelihood estimation in state space models. International Journal of Mathematical Modelling and Numerical Optimisation, 1(1):101-120, 2009.

R. F. Engle. Autoregressive conditional heteroscedasticity with estimates of the variance of United Kingdom inflation. Econometrica, 50(4):987-1007, 1982. 
W.M. Fong and K.H. See. Modelling the conditional volatility of commodity index futures as a regime switching process. Journal of Applied Econometrics, 16(2):133-163, 2001.

A. E. Gelfand and D. K. Dey. Bayesian model choice: Asymptotics and exact calculations. Journal of the Royal Statistical Society Series B, 56(3):501-514, 1994.

S. H. Kang, S. M. Kang, and S. M. Yoon. Forecasting volatility of crude oil markets. Energy Economics, 31(1):119-125, 2009.

L. Kilian. Not all oil price shocks are alike: Disentangling demand and supply shocks in the crude oil market. American Economic Review, 99(3):1053-1069, 2009.

S. Kim, N. Shepherd, and S. Chib. Stochastic volatility: Likelihood inference and comparison with ARCH models. Review of Economic Studies, 65(3):361-393, 1998.

G. Koop. Bayesian Econometrics. Wiley \& Sons, New York, 2003.

S. J. Koopman and E. Hol Uspensky. The stochastic volatility in mean model: Empirical evidence from international stock markets. Journal of Applied Econometrics, 17(6): 667-689, 2002.

D. P. Kroese and J. C. C. Chan. Statistical Modeling and Computation. Springer, New York, 2014.

K. Larsson and M. Nossman. Jumps and stochastic volatility in oil prices: Time series evidence. Energy Economics, 33(3):504-514, 2011.

M. Manera, M. Nicolini, and I. Vignati. Modelling futures price volatility in energy markets: Is there a role for financial speculation? Energy Economics, 2014. Forthcoming.

C. F. Mason and N. A. Wilmot. Jump processes in natural gas markets. Energy Economics, 46(supplement 1):S69-S79, 2014.

H. Mohammadi and L. Su. International evidence on crude oil price dynamics: Applications of ARIMA-GARCH models. Energy Economics, 32(5):1001-1008, 2010.

N. Nomikos and K. Andriosopoulos. Modelling energy spot prices: Empirical evidence from NYMEX. Energy Economics, 34(4):1153-1169, 2012.

G. Peersman and I. Van Robays. Cross-country differences in the effects of oil shocks. Energy Economics, 34(5):1532-1547, 2012.

R .Y. Rubinstein. Optimization of computer simulation models with rare events. European Journal of Operational Research, 99:89-112, 1997.

R. Y. Rubinstein and D. P. Kroese. The Cross-Entropy Method: A Unified Approach to Combinatorial Optimization Monte-Carlo Simulation, and Machine Learning. Springer-Verlag, New York, 2004. 
P. Sadorsky. Stochastic volatility forecasting and risk management. Applied Financial Economics, 15(2):121-135, 2005.

P. Sadorsky. Modeling and forecasting petroleum futures volatility. Energy Economics, 28(4):467-488, 2006.

S. J. Taylor. Modelling stochastic volatility: A review and comparative study. Mathematical Finance, 4(2):183-204, 1994.

A. B. Trolle and E. S. Schwartz. Unspanned stochastic volatility and the pricing of commodity derivatives. Review of Financial Studies, 22(11):4423-4461, 2009.

M. T. Vo. Regime-switching stochastic volatility: Evidence from the crude oil market. Energy Economics, 31(5):779-788, 2009.

J. Yu. Forecasting volatility in the New Zealand stock market. Applied Financial Economics, 12(3):193-202, 2002. 